was $3.9 \%(n=5)$ in the 2 week wait (2WW) pathway versus $7.5 \%(n=11)$ in the routine referral pathway. This may reflect patients being referred on a routine pathway due to reassurance from investigations in the preceding year.

The post endoscopy cancer rate in the oesophagus, stomach and duodenum were $6.5 \% \quad(n=9), 6.4 \% \quad(n=6)$ and $2.3 \%$ $(n=1)$ respectively. The highest rate by individual location was $18.2 \%(n=2)$ in the upper third of the oesophagus. $40 \%$ of post endoscopy cancers showed an alternate diagnosis at initial endoscopy; Gastritis/Oesophagitis $(n=4)$, Hiatus hernia $(n=2)$, Schatzki ring $(n=1)$.

This group also showed a higher TNM staging compared to the background population; pT 3.30 vs. 2.85 ( $\mathrm{p}=0.16, \mathrm{z}$ $=1.41), \mathrm{pN} 2.00$ vs. $1.12(\mathrm{p}=0.17, \mathrm{z}=2.37)$, although this did not reach statistical significance.

Conclusion We have a low rate of delay in diagnosis of our UGI cancers, but it is associated with a higher TNM stage which could impact patient prognosis. A large number of cancers were missed on endoscopy, in particular in the upper third of the oesophagus. Endoscopists should take care to obtain clear views on extubation. A large proportion of patients re-present within one year of endoscopy, so clinicians should re-investigate if there are persistent symptoms.

\section{PTH-026 ROBOSCOPE - 2 CENTRE INITIAL EXPERIENCE}

${ }^{1}$ Stephen Hearing*${ }^{*}{ }^{2}$ Jonathan Fletcher. ${ }^{1}$ Royal Derby Hospital, Derby, UK; ${ }^{2}$ Borders General Hospital, Melrose, UK

\subsection{6/gutjnl-2019-BSGAbstracts.51}

Introduction Robotic colonoscopy was introduced to UK in 2015. It has previously been used in other European centres. Robotic colonoscopy is an automatic self-propelled, user guided, single use colonoscope which has been developed to enable successful caecal intubation, with reduced discomfort (painless colonoscopy).

Methods The technique has been piloted in a number of UK centres, results are presented for initial use in 2 centres.

Results Royal Derby Hospital

10 procedures undertaken: 1 was abandoned because of technical problems with the equipment. Of the 9 procedures undertaken, 4 were successful to the caecum, 5 were unsuccessful (3 to hepatic flexure, 2 to sigmoid). Of the 5 that were unsuccessful, all were changed to traditional colonoscopy and successful colonoscopy to the caecum was performed.

Borders General Hospital

17 procedures undertaken: 9 were successful to the caecum, 8 were unsuccessful (4 to hepatic flexure, 4 to sigmoid). Of the 8 that were unsuccessful, all were changed to traditional colonoscopy and successful colonoscopy to the caecum was performed.

Combined data

Caecal Intubation rate $48 \%$

Mean Procedure time 74 minutes (23 patients)

Analgesia requirements - 26 patients required opiate and midazolam, 1 patient Entonox only

Conclusions In the 2 centres trialling the Robotic colonoscopy, the technique is associated with low caecal intubation rate, long procedure times and analgesia requirements equivalent to traditional colonoscopy. Both units no longer carry out this procedure.

\section{PTH-027 IMPACT OF CHANGES IN GUIDANCE ON VARICEAL PROPHYLAXIS ON RATE OF VARICEAL HAEMORRHAGE IN GLOUCESTERSHIRE}

Melanie Cuffe*, Phoebe Hodges, Victoria Goodall, Coral Hollywood. Gloucestershire Hospitals NHS Foundation Trust, Cheltenham/Gloucester, UK

\subsection{6/gutjnl-2019-BSGAbstracts.52}

Introduction Oesophageal varices develop as a consequence of portal hypertension in patients with cirrhosis and account for around $10 \%$ of admissions with acute GI bleeding in the UK with a significant in-hospital mortality rate of $15 \% .^{1}$ In $2015-$ 2016 the BSG and NICE published new guidance recommending endoscopic variceal band ligation for primary prevention of bleeding. ${ }^{2}{ }^{3}$ A subsequent change in local policy sees patients with grade 2 or 3 varices identified during elective upper GI (UGI) endoscopy offered endoscopic variceal band ligation routinely. We aimed was to assess the impact of this policy change on the rate of variceal haemorrhages.

Methods Electronic endoscopy records at Gloucestershire Hospitals NHS Foundation Trust were interrogated to identify procedures performed for variceal surveillance (VS) or UGI bleeding and where the diagnosis was variceal bleeding over two periods: Jan-May 2015 and Oct 2017 to Mar 2018.

Results Of the 92 VS procedures performed in the first period, 53 had varices, of which 9 (17\%) underwent prophylactic banding.

Of the 246 VS procedures performed in the second period, 166 had varices, of which $52(31.3 \%)$ underwent prophylactic banding.

The proportionate number of variceal bleeds in the first and second period was $8.8 \% \quad(n=10)$ and $6 \% \quad(n=17)$ respectively.

Conclusions The increase in prophylactic banding since the policy change appears to have reduced the proportionate number of variceal bleeds by $2.6 \%$. This is seen alongside a significantly increased number of patients attending for VS, for unclear reasons. This could simply reflect the increasing burden of chronic liver disease. ${ }^{4}$ However, given the short interval period it could reflect an improvement in identifying patients suitable for variceal screening.

\section{REFERENCES}

1. Jairath $\mathrm{V}$, et al. Acute variceal haemorrhage in the United Kingdom: patient characteristics, management and outcomes in a nationwide audit. Dig Liver Dis 2014:46:419-26.

2. Tripathi $D$, et al. UK guidelines on the management of variceal haemorrhage in cirrhotic patients. Gut 2015;0:1-25.

3. Cirrhosis in over 16s: assessment and management. NICE [NG50].

4. Pimpin $L$, et al. Burden of liver disease in Europe: Epidemiology and analysis of risk factors to identify prevention policies. J Hepatol 2018;69(3):718-35.

\section{PTH-028 HIGHER-QUALITY COLON CLEANSING IMPROVES LESION DETECTION DURING COLONOSCOPY: A QUANTITATIVE ANALYSIS OF PHASE 3 TRIALS}

${ }^{1}$ Andrew Holgate*, 'Soniya Mokashi, ${ }^{2} J o n a t h a n$ Manning. 'Norgine Pharmaceuticals Ltd, Uxbridge, UK; ${ }^{2}$ Borders General Hospital, Melrose, UK

\subsection{6/gutjnl-2019-BSGAbstracts.53}

Introduction Colon cleansing is important for reliable lesion detection during colonoscopy, however the quantitative benefits of improved cleansing quality remain poorly understood. This post hoc analysis of three similarly designed phase 3 
randomised clinical trials assessed the treatment-independent trends for overall colon cleansing quality versus overall colon lesion detection.

Methods Three multi-centre phase 3 clinical trials compared the cleansing efficacy and safety of the 1L PEG NER1006 $\left(\right.$ PLENVU $\left.^{\oplus}\right)$ versus standard bowel preparations in patients aged 18-85 years. Treatment-blinded assessment of colon cleansing quality was performed by both site colonoscopists (SC; who also detected all lesions per local practice) and by central readers (CR). Two validated cleansing scales were used: the Harefield Cleansing Scale (HCS) and the Boston Bowel Preparation Scale (BBPS; only by CR). Patients with documented HCS cleansing grades D-A, overall BBPS scores $0-9$, and overall colon lesion counts were included in this analysis. Logistic regression trends were fitted to polyp- (PDR) and adenoma (ADR) detection rates, using cleansing quality as a covariate.

Results Out of 1,985 randomised patients, 1,749 patients were included (table 1). With site colonoscopists' HCS grades, the logistic regression for relative lesion detection demonstrated an odds ratio of 1.17 , i.e. for each incremental increase in the HCS colon cleansing grade from $\mathrm{D}$ to $\mathrm{A}$ there was a 1.17 times increase in both PDR $(\mathrm{P}=0.009)$ and $\operatorname{ADR}(\mathrm{P}=0.019)$. With central readers, the corresponding increase was 1.24 times for PDR $(\mathrm{P}=0.005)$ and 1.26 times for ADR $(\mathrm{P}=0.006)$. With central readers, each incremental increase in the successful BBPS scores 6-9 resulted in a 1.08 times increase in PDR $(\mathrm{P}<0.001)$ and 1.10 times increase in $\operatorname{ADR}(\mathrm{P}<0.001)$ versus failing BBPS scores 0-5.

Conclusions Adopting a better bowel preparation remains a good way to improve quality in colonoscopy. With both HCS and BBPS, an increased overall colon cleansing quality was associated with greater overall colon PDR and ADR across the full range from cleansing failures and up to high-quality cleansing.

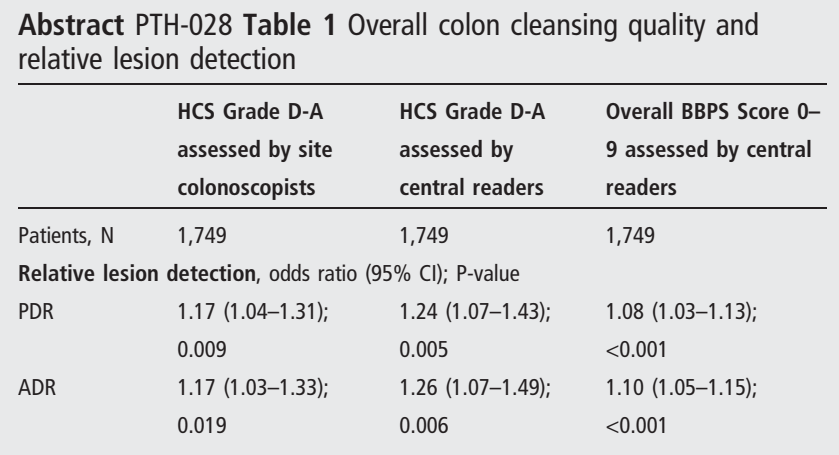

\section{PTH-029 CLOSURE OF GASTROCUTANEOUS FISTULAS WITH AN OVER-THE-SCOPE-CLIP (OTSC) - A LARGE TERTIARY HOSPITAL EXPERIENCE}

Thomas Hollingworth*, Philip Boger, Emily Clarke, Praful Patel, Charlotte Rutter, Trevor Smith, Imdadur Rahman. University Hospital Southampton, Southampton, UK

\subsection{6/gutinl-2019-BSGAbstracts.54}

Introduction Gastrocutaneous fistulae (GF) are a rare, but difficult to manage complication after percutaneous endoscopic gastrostomy (PEG) tube removal. In cases, refractory to conventional medical management various approaches have been used for their treatment including; surgical closure, through the scope clipping and endoscopic suturing with variable results. The over-the-scope-clip (OTSC) has an emerging role in the endoscopic closure of gastrointestinal wall defects, including persistent GF. The aim of this study was to assess the efficacy and safety of the use of OTSC for the closure of persistent GF.

Methods A prospectively kept database was analysedfrom September 2016 to January 2019 was undertaken of all persistent GF using an OTSC 12/6 GF clip. Prior to the deployment of an OTSC, the fistulous tract was disrupted using a wire brush, which also acted as a guide for placement. The use of ancillary techniques such as snare debulking or knife resection was utilised if needed. Primary outcome measures were; successful deployment of the OTSC, procedure time, complications and 30 day success. Secondary outcome measures were PEG dwell time prior to removal, time from diagnosis to closure and sedation type.

Results A total of 22 procedures were performed, on 12 male \& 10 female patients with a mean age of 47 . Median PEG dwell time was 16 months prior to removal. 64\% of the procedures were performed with conscious sedation, with $36 \%$ requiring enhanced sedation with anaesthetic support. Technical success was $100 \%$, with 2 procedures requiring ancillary techniques to facilitate the deployment of the OTSC, resulting in 1 complication of intra-procedural bleeding (stopped endoscopically). The median procedure time was 14 mins (range 9-35). A 30 \& 90 day success was reported in $96 \%$ cases, with only one fistula recurring.

Discussion This is the largest cohort of patients with a persistent GF treated with an OTSC. This evolving procedure is an effective and safe method for the treatment of persistent GF. It has a much lower morbidity compared to surgery and shorter procedure times than more invasive endoscopic treatment options, such as endoscopic suturing.

\section{PTH-030 OUTCOMES OF HEMOSPRAY USE IN PEPTIC ULCER UPPER GASTROINTESTINAL BLEEDS: OUTCOMES FROM THE HEMOSPRAY REGISTRY}

\begin{abstract}
${ }^{1,2}$ Mohamed Hussein*, ${ }^{2}$ Durayd Alzoubaidi, ${ }^{3}$ Miguel Fraile, ${ }^{3}$ Jacobo Ortiz Fernandez-sordo, ${ }^{3}$ Krish Ragunath, ${ }^{4}$ Radu Rusu, ${ }^{4}$ John Dunn, ${ }^{14}$ Johannes Rey, ${ }^{5}$ Shraddha Gulati, ${ }^{5}$ Bu'Hussain Hayee, ${ }^{6}$ Selena Dixon, ${ }^{6}$ Sulleman Moreea, ${ }^{7}$ Duncan Napier, ${ }^{7} J o h n$ Anderson, ${ }^{8}$ Martin Dahan, ${ }^{9}$ Max Hu, ${ }^{9}$ Patricia Duarte, ${ }^{9}$ Phil Boger, ${ }^{10}$ John Mcgoran, ${ }^{10}$ Inder Mainie, ${ }^{11}$ Alberto Murino, ${ }^{11}$ Sina Jameie- oskooei, ${ }^{11} \mathrm{Edward}$ Despott, ${ }^{12}$ Cora Steinheber, ${ }^{12}$ Martin Goetz, ${ }^{13}$ Sharmila Subramaniam, ${ }^{13}$ Pradeep Bhandari, ${ }^{1,2}$ Laurence Lovat, ${ }^{8}$ Emmanuel Coron, ${ }^{14}$ Ralf Keisslich, ${ }^{1,2}$ Rehan Haidry. ${ }^{1} U C L H, U K ;{ }^{2} U C L$, UK; ${ }^{3}$ Nottingham Digestive Diseases Centre, UK; ${ }^{4}$ Guys and St Thomas Hospitals, UK; ${ }^{5}$ Kings college Hospital, UK; ${ }^{6}$ Bradford Teaching Hospital NHS Trust, UK; ${ }^{7}$ Gloustershire Hospitals NHS Foundation Trust, UK; ${ }^{8}$ University Hospital of Nantes, France; ${ }^{9}$ University Hospital Southampton, UKi ${ }^{10}$ Belfast NHS Trust, UK; ${ }^{11}$ Royal Free Hospital, UK; ${ }^{12}$ Tübingen University Hospital,UK; ${ }^{13}$ University of Portsmouth, UK; ${ }^{14}$ Horst Schmidt Kliniken, Germany
\end{abstract}

\subsection{6/gutjnl-2019-BSGAbstracts.55}

Introduction Peptic ulcers are the commonest cause of upper Gastrointestinal bleeding (UGIB). Hemospray (Cook Medical, North Carolina, USA) is a novel haemostatic powder aimed to treat UGIB. The aim of this study is to look at outcomes in patients with peptic ulcer GI bleeds treated with hemospray in 13 centres.

Methods Data was prospectively collected on hemospray use in UGIBs in the UK, France and Germany (Jan'16-Sept'18). Hemospray was used for peptic ulcer UGIBs as a 